\title{
LMI-Based Fuzzy Optimal Variance Control of Airfoil Model Subject to Input Constraints
}

\author{
Sean S.M. Swei \\ Research Scientist \\ Intelligent Systems Division \\ NASA Ames Research Center \\ Moffett Field, California 94035 \\ Email: sean.s.swei@nasa.gov
}

\author{
Mohammad A. Ayoubi \\ Associate Professor \\ Mechanical Engineering Department \\ Santa Clara University \\ Santa Clara, California 95053 \\ Email:maayoubi@scu.edu
}

\begin{abstract}
This paper presents a study of fuzzy optimal variance control problem for dynamical systems subject to actuator amplitude and rate constraints. Using Takagi-Sugeno fuzzy modeling and dynamic Parallel Distributed Compensation technique, the stability and the constraints can be cast as a multi-objective optimization problem in the form of Linear Matrix Inequalities. By utilizing the formulations and solutions for the input and output variance constraint problems, we develop a fuzzy fullstate feedback controller. The stability and performance of the proposed controller is demonstrated through its application to the airfoil flutter suppression.
\end{abstract}

\section{INTRODUCTION}

Control of dynamical systems with bounded control input has been studied for decades [1]-[5]. In practical applications, it has been shown that the actuator rate limit is the primary source of instability and performance degradation, especially for aircraft control systems, e.g. see [2] and [4]. Previous works on bounded input and input rate; see [1]-[3] and the references therein, offer a good framework for control implementation. A simple "software limiter" idea was proposed in Ref. [2]. There, by introducing the derivative and limited integration blocks, a commanded input signal can be re-generated which satisfies both input and input rate constraints. In Ref. [3] a bounded input and input rate problem description was given that, to an extent, prescribes how the actuator should behavior. An extended high performance bounded control algorithm was proposed in Ref. [1], which was used to suppress the vibrational motion of the aircraft wing subject to bounded actuation on position/rate. All these results were obtained by considering only one given dynamical system description. For a control system that contains scenario dependent (varying) parameters, multiple system descriptions can be generated based on each parameter value. In this case, one common approach that is used to address the gain scheduling and stability/performance issues is the Linear Parameter Varying (LPV) method [6], [7]. In the past three decades, the theory of fuzzy control systems has been developed tremendously. In particular, the stability and performance of a fuzzy control problem can be cast as solving a feasibility problem in the form of Linear Matrix Inequalities (LMIs) [8]-[11]. Therefore, the numerically efficient LMI-based convex optimization tools and algorithms can readily be utilized for control system analysis and synthesis.

In this paper, we consider the problem of fuzzy optimal variance control for a parameter varying (nonlinear) "smart" airfoil model [12], as shown in Fig. 1 where the actuator amplitude and rate are constrained. The Takagi-Sugeno (TS) fuzzy model is used to approximate the parameter varying system via a set of convex combination of local linear models. We show that the bounded input and bounded input rate problem can be converted into a bounded output and bounded input problem by augmenting the control input as an additional state. We show that the T-S fuzzy optimal variance control problem is closely related to the input and output variance constrained problems [13], whose formulations and solutions are essential in attaining the main results of this paper. We present that the feasibility of the problem via a full-state feedback controller can be characterized using the LMIs for each local model.

\section{PROBLEM STATEMENT}

We consider a 2-D smart airfoil model as illustrated in Fig. 1. The airfoil is subjected to the free stream airflow at varying speed $V$, and is allowed to pitch $(\alpha)$ and plunge (h) about the supported axis. The rotational and translational spring constants are denoted by $K_{\alpha}$ and $K_{h}$, respectively, as shown in Fig. 1. A light ballast mass, $m$, which is placed along the airfoil chord, is electronically activated and served as controllable effector. The objective of this smart airfoil platform is to suppress the fluttering motion of the airfoil, subjected to varying free stream airspeed, through actuation of the ballast mass. However, the ballast motion is physically constrained by the length of its travel and the motor driving rate, and these constraints pose a practical control design challenge.

The equations of motion for this 2-D smart airfoil model can be described in the nondimensional form as follows [12],

$$
\begin{aligned}
& {\left[\begin{array}{cc}
1+\beta & \bar{x}_{\alpha} \\
\bar{x}_{\alpha} & \bar{r}_{\alpha}^{2}
\end{array}\right]\left[\begin{array}{c}
\ddot{\bar{h}}(\tau) \\
\ddot{\alpha}(\tau)
\end{array}\right]+\left[\begin{array}{cc}
2 \bar{V} / \mu & 0 \\
-2 \bar{V} \bar{e} / \mu & 0
\end{array}\right]\left[\begin{array}{c}
\dot{\bar{h}}(\tau) \\
\dot{\alpha}(\tau)
\end{array}\right]+} \\
& {\left[\begin{array}{cc}
\omega_{h}^{2} / \omega_{\alpha}^{2} & 2 \bar{V}^{2} / \mu \\
0 & -2 \bar{V}^{2} \bar{e} / \mu+\bar{r}_{\alpha}^{2}
\end{array}\right]\left[\begin{array}{c}
\bar{h}(\tau) \\
\alpha(\tau)
\end{array}\right]=\left[\begin{array}{c}
0 \\
\beta \bar{g}
\end{array}\right]\{\bar{y}(\tau)+d(\tau)\}}
\end{aligned}
$$




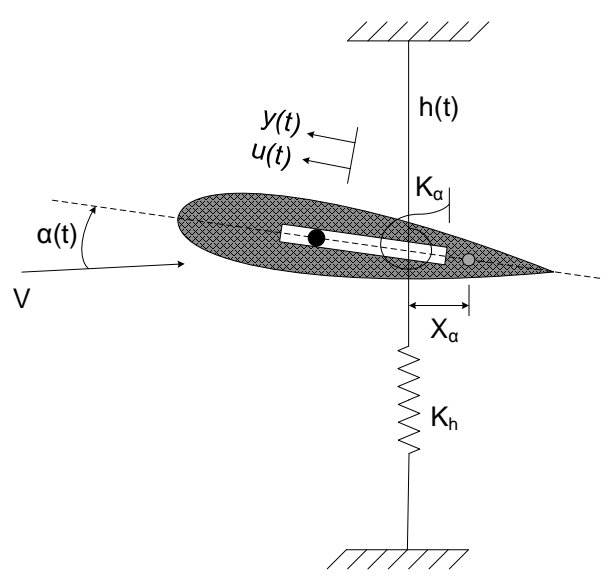

Fig. 1. The smart airfoil model

where $\tau=\omega_{\alpha} t$ denotes the nondimensional time, $\beta$ the mass ratio between $m$ and the mass of airfoil, $b$ the airfoil semichord, $\bar{V}=V / \omega_{\alpha} b$ the nondimensional airspeed, $\bar{h}=h / b$ the nondimensional plunging displacement, $\bar{y}=y / b$ the nondimensional displacement, and $d(\tau)$ the nondimensional random disturbance input. Now, if we let $x=\left[\begin{array}{lll}\bar{h} \dot{\bar{h}} \alpha \dot{\alpha}\end{array}\right]$, then (1) can be rewritten in the state-space form as follows,

$$
\Sigma_{\bar{V}}: \quad \dot{x}(\tau)=A(\bar{V}) x(\tau)+B u(\tau)+D d(\tau)
$$

where

$$
\begin{gathered}
A(\bar{V})=a\left[\begin{array}{cc}
0 & 1 / a \\
-\left(\omega_{n} / \omega_{\alpha}\right)^{2} \bar{r}_{\alpha}^{2} & -\frac{2 \bar{V}}{\mu}\left(\bar{r}_{\alpha}^{2}+\bar{x}_{\alpha} \bar{e}\right) \\
0 & 0 \\
\left(\omega_{n} / \omega_{\alpha}\right)^{2} \bar{x}_{\alpha} & \frac{2 \bar{V}}{\mu}\left[\bar{x}_{\alpha}+(1+\beta) \bar{e}\right] \\
0 & 0 \\
-\frac{2 \bar{V}^{2}}{\mu}\left(\bar{r}_{\alpha}^{2}+\bar{x}_{\alpha} \bar{e}\right)+\bar{x}_{\alpha} \bar{r}_{\alpha}^{2} & 0 \\
0 & 1 / a \\
B=\left[\begin{array}{c}
\frac{2 \bar{V}^{2}}{\mu}\left[\bar{x}_{\alpha}+(1+\beta) \bar{e}\right]-(1+\beta) \bar{r}_{\alpha}^{2} \\
0 \\
-a \bar{x}_{\alpha} \beta \bar{g} \\
0 \\
a(1+\beta) \beta \bar{g}
\end{array}\right], D=B,
\end{array}\right.
\end{gathered}
$$

and $a=1 /\left[(1+\beta) \bar{r}_{\alpha}^{2}-\bar{x}_{\alpha}^{2}\right]$. Since $A(\bar{V})$ is explicitly function of free stream airspeed, the dynamic response of $\Sigma_{\bar{V}}$ varies as function of $\bar{V}$. In addition, the control input $u(\tau)$ is bounded by the length of groove and $\dot{u}(\tau)$ is constrained by the allowable motor torque. More specifically, in this study, we consider that $u=\left[u_{1}, u_{2}, \cdots u_{m}\right]$ and $\dot{u}=\left[\dot{u}_{1}, \dot{u}_{2}, \cdots \dot{u}_{m}\right]$, and

$$
\left|u_{i}\right| \leq \sigma_{i},\left|\dot{u}_{i}\right| \leq \tau_{i}, \quad(i=1, \ldots, m)
$$

where $\left(\sigma_{i}, \tau_{i}\right)$ are known constants. Let the $\mathscr{L}_{2}$-norm of the random disturbance $d(\cdot)$ be given by

$$
\|d\|_{2}^{2} \triangleq \int_{0}^{\infty} d^{T}(\tau) d(\tau) d \tau
$$

and the weighted $\mathscr{L}_{2}$ disturbance set $\mathscr{W}$ be defined as

$$
\mathscr{W} \triangleq\left\{d: \mathbb{R} \rightarrow \mathbb{R}^{d},\left\|W^{\frac{1}{2}} d\right\|_{2}^{2} \leq 1\right\}
$$

where $W$ is a positive definite symmetric matrix. Let the $\mathscr{L}_{\infty}$ norm of $y(\cdot)$ be defined by

$$
\|y\|_{\infty}^{2} \triangleq \sup _{\tau \geq 0} y^{T}(\tau) y(\tau) .
$$

Therefore, the input amplitude and rate constraints given in (4) are precisely the $\mathscr{L}_{\infty}$ bounds.

\section{TAKAGI-SUGENO FUZZY MODEL}

The power of T-S fuzzy modeling technique is that the nonlinear dynamical systems can be expressed as a convex combination of local linear systems through fuzzy IFTHEN rules which represent local input-output relations. It has been shown in [15] that smooth nonlinear systems can be adequately approximated by T-S fuzzy models. Before building a T-S fuzzy model, we first need to determine the premise variables and develop the corresponding model rules. Let $\theta(t)$ denote a vector of $p$ known premise variables, i.e. $\theta(t)=\left[\theta_{1}(t), \theta_{2}(t), \ldots, \theta_{p}(t)\right]$, then the number of model rules $r$ is $2^{p}$. For the purpose of this paper, we assume that $\theta$ is independent of system states and inputs. To this end, we may apply the above approximation to create a T-S fuzzy model for $\Sigma_{\bar{V}}$ and obtain a local linear model for each fuzzy rule as follows.

Model Rule $i$ :

IF $\theta_{1}(t)$ is $\Xi_{i 1}, \ldots, \theta_{p}(t)$ is $\Xi_{i p}$, THEN:

$$
\Sigma_{i}:\left\{\begin{array}{l}
\dot{x}(t)=A_{i} x(t)+B_{i} u(t)+D_{i} d(t) \quad(i=1,2, \ldots, r) \\
y(t)=C_{i} x(t)
\end{array}\right.
$$

where $\Xi_{i j}$ is the fuzzy set, $y$ the controlled output, and $\left(A_{i}, B_{i}, C_{i}, D_{i}\right)$ are known constant matrices. We assume that the pair $\left(A_{i}, B_{i}\right)$ is controllable. In addition, recall that each entry of $u$ and $\dot{u}$ is bounded by (4).

The firing strength of each model rule can be determined using $T$ - norm product [11] as follows,

$$
w_{i}(\theta)=\prod_{j=1}^{p} \Xi_{i j}(\theta)
$$

and the fuzzy basis functions $h_{i}(\theta)$ are determined by

$$
h_{i}(\theta)=\frac{w_{i}(\theta)}{\sum_{i=1}^{r} w_{i}(\theta)} ; \sum_{i=1}^{r} h_{i}(\theta)=1, h_{i}(\theta) \geq 0 .
$$

After combining all the rules of T-S models, the nonlinear system $\Sigma_{\bar{V}}$ can be approximated by convex combination of local models as

$$
\Sigma_{T S}:\left\{\begin{array}{l}
\dot{x}=\sum_{i=1}^{r} h_{i}(\theta)\left\{A_{i} x+B_{i} u+D_{i} d\right\} \\
y=\sum_{i=1}^{r} h_{i}(\theta) C_{i} x
\end{array}\right.
$$

Now, the Full State Fuzzy Optimal Variance Control Problem for $\Sigma_{\bar{V}}$ can be stated as follows:

Given the input amplitude and rate constraints as in (4) and the initial condition constraint $\|x(0)\| \leq \phi$, find a fuzzy full-state feedback controller so that it guarantees: i) internal stability for $\Sigma_{T S}$; ii) minimum output $\mathscr{L}_{\infty}$ performance (variance) cost. 
In this paper, we introduce a convenient way of incorporating the effect of $\dot{u}$ by augmenting it into the system description, and this approach has been adopted throughout the paper. We introduce an augmented system description for $i$ th model rule $\Sigma_{i}$ as follows,

$$
\Sigma_{a i}:\left\{\begin{array}{l}
\dot{x}_{a}=A_{a i} x_{a}+B_{a} u_{a}+D_{a i} d \quad(i=1,2, \ldots, r) \\
y_{a}=C_{a i} x_{a}
\end{array}\right.
$$

where

$$
\begin{aligned}
& x_{a}=\left[\begin{array}{c}
x \\
u
\end{array}\right], u_{a}=\dot{u}, A_{a i}=\left[\begin{array}{cc}
A_{i} & B_{i} \\
0 & 0
\end{array}\right], B_{a}=\left[\begin{array}{c}
0 \\
I_{m}
\end{array}\right], \\
& D_{a i}=\left[\begin{array}{c}
D_{i} \\
0
\end{array}\right], y_{a}=\left[\begin{array}{c}
y \\
u
\end{array}\right], C_{a i}=\left[\begin{array}{cc}
C_{i} & 0 \\
0 & I_{m}
\end{array}\right] .
\end{aligned}
$$

Note that the new control input to the augmented system is the input rate $\dot{u}$. Furthermore, the input amplitude and rate constraints in (5) have now become the output and input constraints in the augmented system (9). Similarly, we can define the augmented T-S model for $\Sigma_{T S}$ as follows,

$$
\Sigma_{T S a}:\left\{\begin{array}{l}
\dot{x}_{a}=\sum_{i=1}^{r} h_{i}(\theta)\left\{A_{a i} x_{a}+B_{a} u_{a}+D_{a i} d\right\} \\
y_{a}=\sum_{i=1}^{r} h_{i}(\theta) C_{a i} x_{a}
\end{array}\right.
$$

\section{SOME PRELIMINARY RESULTS}

In this section, we present some preliminary results on LMI characterization for the input and output variance constrained problems. These results will play an instrumental role in obtaining the main theorem of this paper. We consider the following linear systems,

$$
\left\{\begin{array}{l}
\dot{x}(t)=A x(t)+D d(t) \\
y_{i}(t)=C_{i} x(t) \quad\left(i=1,2, \ldots, k_{c}\right)
\end{array}\right.
$$

where $\left(C_{i}, A, D\right)$ are matrices of compatible dimensions and $d \in \mathscr{W}$. It is well understood that, if $A$ is Hurwitz, the output variance for (11) is given by [16]

$$
Y_{i}=C_{i} X C_{i}^{T},\left(i=1,2, \ldots, k_{c}\right),
$$

where $X>0$ is the controllability Gramian matrix satisfying the following Lyapunov equation

$$
X A^{T}+A X+D W D^{T}=0 .
$$

Therefore, for any $d \in \mathscr{W}$, the $\mathscr{L}_{\infty}$-norm of the output $y_{i}$ is given by [17]

$$
\left\|y_{i}\right\|_{\infty}^{2} \leq \sigma_{\max }\left(Y_{i}\right)
$$

where $\sigma_{\max }(\cdot)$ denotes the maximum singular value. This indicates that the worst case output, $\left\|y_{i}\right\|_{\infty}$, is bounded by the squared root of the maximum singular value of output variance. Furthermore, given the desired output variance $\bar{Y}_{i}$, the output variance constrained problem for (11) is said to be solved when

$$
Y_{i} \leq \bar{Y}_{i}\left(i=1,2, \ldots, k_{c}\right) .
$$

The following lemma completely characterizes the output variance constrained problem using the LMIs [13].
Lemma 1: Consider (11) and let $\bar{Y}_{i}>0, i=1,2, \ldots, k_{c}$, be the desired output variance. Then, the output variance constrained problem is solvable, if there exists $\bar{X}>0$ satisfying

$$
\begin{aligned}
& \text { (i) }\left[\begin{array}{cc}
\bar{X} A^{T}+A \bar{X} & D W^{\frac{1}{2}} \\
W^{\frac{1}{2}} D^{T} & -I
\end{array}\right]<0, \\
& \text { (ii) } C_{i} \bar{X} C_{i}^{T}<\bar{Y}_{i}, \quad\left(i=1,2, \ldots, k_{c}\right) .
\end{aligned}
$$

Next, we present the input variance constraint control problem [13]. To study this problem, we consider the following linear control systems,

$$
\left\{\begin{array}{l}
\dot{x}(t)=A x(t)+B u(t)+D d(t) \\
y_{i}(t)=C_{i} x(t),\left(i=1,2, \ldots, k_{c}\right)
\end{array}\right.
$$

where the pair $(A, B)$ is assumed controllable, and $d \in \mathscr{W}$. We consider the full state feedback control law of the form

$$
u(t)=G x(t)
$$

such that $A_{c l}=(A+B G)$ is Hurwitz. Then, the input variance $U$ for $u(t)$ is given by [13]

$$
U=G X G^{T}
$$

where $X \geq 0$ solves the following Lyapunov equation

$$
A_{c l} X+X A_{c l}^{T}+D W D^{T}=0 .
$$

Note that the control input $u(t)$ can be partitioned into $u=$ $\left[u_{1}, u_{2}, \cdots, u_{m}\right]^{T}$, and each $u_{i}$ can be expressed as

$$
u_{i}(t)=K_{i} x(t), G=\left[\begin{array}{llll}
K_{1} & K_{2} & \cdots & K_{m}
\end{array}\right]^{T} .
$$

The $\mathscr{L}_{\infty}$-norm of input $u_{i}$ is given by

$$
\left\|u_{i}\right\|_{\infty}^{2} \leq K_{i} X K_{i}^{T}=\left\{G X G^{T}\right\}_{i i},(i=1,2, \ldots, m)
$$

where \{\}$_{i i}$ denotes the $i$ th diagonal entry. If we define $U_{i}=$ $\left\{G X G^{T}\right\}_{i i}$ to be the $i$ th input variance, then from (22) we obtain

$$
\left\|u_{i}\right\|_{\infty} \leq \sqrt{U_{i}}, \quad(i=1,2, \ldots, m)
$$

that is, the maximum input is bounded by the squared root of input variance. Let $\bar{U}_{i}>0, i=1,2, \ldots, m$, be the given input variance for $u_{i}(t)$. Then, the input variance constraint control problem is solvable via a static full-state feedback controller of the form (21), if it minimizes the output variance cost

$$
J=\operatorname{trace}\left\{C_{j} X C_{j}^{T}\right\}, \quad\left(j=1,2, \ldots, k_{c}\right),
$$

subject to: 1) $A_{c l}=(A+B G)$ is Hurwitz, and 2) $U_{i}=K_{i} X K_{i}^{T} \leq$ $\bar{U}_{i}, i=1,2, \ldots, m$, where $X \geq 0$ solves (20). The next lemma characterizes the input variance constraint control problem using LMIs, whose proof follows readily from Lemma 1.

Lemma 2: Consider (17) and let $\bar{U}_{i}>0, i=1,2, \ldots, m$, be the given input variance. Then, the input variance constraint control problem via a static full-state feedback control is solvable, if there exist a matrix $F$ and a positive definite symmetric matrix $\bar{X}$ that minimize

$$
\min _{\bar{X}, F} \operatorname{trace}\left\{C_{j} \bar{X} C_{j}^{T}\right\},\left(j=1,2, \ldots, k_{c}\right),
$$


subject to the following LMIs,

(i)

$$
\left[\begin{array}{cc}
\left(\bar{X} A^{T}+A \bar{X}+F^{T} B^{T}+B F\right) & D W^{\frac{1}{2}} \\
W^{\frac{1}{2}} D^{T} & -I
\end{array}\right]<0,
$$

$$
\left[\begin{array}{cc}
\bar{U}_{i} & \Phi_{i} F \\
F^{T} \Phi_{i}^{T} & \bar{X}
\end{array}\right]>0, \quad(i=1,2, \ldots, m)
$$

where $\Phi_{i}$ is a projection matrix such that $u_{i}=\Phi_{i} u$.

Furthermore, if there exists a feasible solution to the above LMIs, then the full state feedback gain $G=F \bar{X}^{-1}$.

\section{FUZZY OPTIMAL VARIANCE CONTROL PROBLEM: FULL-STATE FEEDBACK}

The Parallel Distributed Compensation technique, which was introduced by Wang et al. [18], is based on the T-S fuzzy model approximation. This model-based fuzzy control structure is derived according to the T-S fuzzy model, therefore each control rule is designed to compensate for each model rule of fuzzy system. Furthermore, it was shown in [18] that the convex combination of model rules can be stabilized by the same convex combination of control rules. In this section, we consider the fuzzy optimal variance control problem for $\Sigma_{T S}$ (or $\Sigma_{T S a}$ ) by utilizing the full-state feedback controllers.

Recall the augmented system description for $i$ th model rule $\Sigma_{a i}$ as described in (9). The proposed fuzzy full-state feedback for $i$ th control rule will have the same premise variables, i.e. "IF" statement, but different consequent, i.e. "THEN" statement. The general structure of each state feedback control rule for $\Sigma_{a i}$ is given below.

Control Rule $i$ :

IF $\theta_{1}(t)$ is $\Xi_{i 1}, \ldots, \theta_{p}(t)$ is $\Xi_{i p}$, THEN:

$$
u_{a}(t)=\dot{u}(t)=-F_{i} x_{a}(t), \quad(i=1,2, \ldots, r)
$$

where $F_{i}$ is the internally stabilizing feedback gain matrix for $i$ th T-S fuzzy model $\Sigma_{a i}$. Since $\left(A_{i}, B_{i}\right)$ is controllable, it is straightforward to show that $\left(A_{a i}, B_{a}\right)$ is also controllable. Hence, there always exists a constant gain matrix $F_{i}$ so that $\left(A_{a i}-B_{a} F_{i}\right)$ is Hurwitz, for $i=1,2, \ldots, r$. Then, the overall control input with fuzzy basis functions becomes

$$
u_{a}(t)=-\sum_{i=1}^{r} h_{i}(\theta) F_{i} x_{a}(t) .
$$

Let $F_{i}$ be partitioned according to $x_{a}(t)$ as

$$
F_{i}=\left[\begin{array}{ll}
F_{x i} & F_{u i}
\end{array}\right] .
$$

Then, it follows from (28) that

$$
\dot{u}(t)=-\sum_{i=1}^{r} h_{i}(\theta)\left\{F_{x i} x(t)+F_{u i} u(t)\right\},
$$

which is an ordinary differential equation in $u(t)$. Since $x(t)$ is available for feedback, $u(t)$ can be solved from (30) with given initial condition $u(0)$. Therefore, while (28) is a static full-state feedback controller for $\Sigma_{T S a}$, it is a dynamic full-state feedback controller for $\Sigma_{T S}$. The following theorem, which utilizes Lemmas 1 and 2, contains the main result.

Theorem 1: Consider the augmented T-S fuzzy system $\Sigma_{T S a}$ described in (10). The fuzzy optimal variance control problem is solvable via a static full-state feedback controller (28), if there exist matrices $S_{i}(i=1,2, \ldots, r)$ and a positive definite symmetric matrix $X$ that minimize the output variance cost

$$
\begin{gathered}
\min _{X, S_{1}, \cdots, S_{r}} \operatorname{trace}\left\{\left(C_{a i}\right)_{j} X\left(C_{a i}\right)_{j}^{T}\right\}, \\
\left(i=1,2, \ldots, r ; j=1,2, \ldots, k_{c}\right)
\end{gathered}
$$

subject to the following LMIs,

(i)

$$
\begin{gathered}
{\left[\begin{array}{cc}
X A_{a i}^{T}+A_{a i} X-S_{i}^{T} B_{a}^{T}-B_{a} S_{i} & D_{a i} W^{\frac{1}{2}} \\
W^{\frac{1}{2}} D_{a i}^{T} & -I
\end{array}\right]<0,} \\
(i=1,2, \ldots, r)
\end{gathered}
$$

(ii)

$$
\left[\begin{array}{cc}
\tau_{j}^{2} & \Phi_{j} S_{i} \\
S_{i}^{T} \Phi_{j}^{T} & X
\end{array}\right] \geq 0, \quad(i=1,2, \ldots, r ; j=1,2, \ldots, m)
$$

(iii)

$$
\left[\begin{array}{ll}
X_{11} & X_{12} \\
X_{12}^{T} & X_{22}
\end{array}\right]-\left[\begin{array}{cc}
\phi^{2} I & 0 \\
0 & 0
\end{array}\right] \geq 0
$$
(iv)

where $X_{i j}$ are partitions of $X$ according to $x_{a}$.

$$
\sigma_{j}^{2}-\Theta_{k} X \Theta_{k}^{T} \geq 0, \quad(j=1,2, \ldots, m ; k=n+j)
$$

where $\left(C_{a i}\right)_{j}$ denotes the $j$ th row of $C_{a i}$, and $\Phi_{k}$ and $\Theta_{k}$ are row vectors of dimension $m$ and $n+m$, respectively, with 1 at $k$ th entry and 0 elsewhere. Furthermore, if a feasible solution exists to the above LMIs, then the fuzzy full-state feedback gain $F_{i}=S_{i} X^{-1}$.

\section{NUMERICAL SIMULATION}

In this section, we demonstrate the efficacy and performance of the proposed fuzzy full-state feedback controller by applying it to the smart airfoil model introduced in Fig. 1. Recall the equations of motion $\Sigma_{\bar{V}}$ described in (2),

$$
\Sigma_{\bar{V}}: \quad \dot{x}(\tau)=A(\bar{V}) x(\tau)+B u(\tau)+D d(\tau) .
$$

Before proceeding to design a T-S fuzzy state-feedback controller, we need to construct a T-S fuzzy model for $\Sigma_{\bar{V}}$. We utilize the method of sector nonlinearity to bound the nonlinear terms in (3) and define the nonlinear terms as follows,

$$
\left\{\begin{array}{l}
z_{1}(\bar{V})=-\frac{2 \bar{V}^{2}}{\mu} a\left(\bar{r}_{\alpha}^{2}+\bar{x}_{\alpha} \bar{e}\right)+a \bar{x}_{\alpha} \bar{r}_{\alpha}^{2} \\
z_{2}(\bar{V})=\frac{2 \bar{V}^{2}}{\mu} a\left[\bar{x}_{\alpha}+(1+\beta) \bar{e}\right]-a(1+\beta) \bar{r}_{\alpha}^{2}
\end{array}\right.
$$

By choosing a range for $\bar{V} \in\left[\begin{array}{ll}\bar{V}_{m} & \bar{V}_{M}\end{array}\right]$, the minimum and maximum values of $z_{1}(\bar{V})$ and $z_{2}(\bar{V})$ can be evaluated at

$$
\left\{\begin{array}{l}
z_{1}(\bar{V})=\Xi_{11}(\bar{V}) z_{1 M}+\Xi_{21}(\bar{V}) z_{1 m} \\
z_{2}(\bar{V})=\Xi_{12}(\bar{V}) z_{2 M}+\Xi_{22}(\bar{V}) z_{2 m}
\end{array}\right.
$$


where $\Xi_{i j}, i, j=1,2$, denote the membership functions, and

$$
\Xi_{1 j}\left(z_{j}\right)=\frac{z_{j}-z_{j m}}{z_{j M}-z_{j m}}, \quad \Xi_{2 j}\left(z_{j}\right)=\frac{z_{j M}-z_{j}}{z_{j M}-z_{j m}}, \quad(j=1,2)
$$

and $z_{1 M}=z_{1}\left(\bar{V}_{M}\right), z_{1 m}=z_{1}\left(\bar{V}_{m}\right), z_{2 M}=z_{2}\left(\bar{V}_{M}\right), z_{2 m}=z_{2}\left(\bar{V}_{m}\right)$.

Using the triangular membership functions and the model parameters listed in Table I, the T-S fuzzy models for $\Sigma_{\bar{V}}$ can be obtained as:

Model Rule 1:

IF $z_{1}(\bar{V})$ is "Positive" and $z_{2}(\bar{V})$ is "Small," THEN:

$\dot{x}(t)=A_{1} x+B u+D d$

Model Rule 2:

IF $z_{1}(\bar{V})$ is "Positive" and $z_{2}(\bar{V})$ is "Big," THEN:

$\dot{x}(t)=A_{2} x+B u+D d$

Model Rule 3:

IF $z_{1}(\bar{V})$ is "Negative" and $z_{2}(\bar{V})$ is "Small," THEN:

$\dot{x}(t)=A_{3} x+B u+D d$

Model Rule 4:

IF $z_{1}(\bar{V})$ is "Negative" and $z_{2}(\bar{V})$ is "Big," THEN:

$\dot{x}(t)=A_{4} x+B u+D d$

where the matrices $A_{i},(i=1, \ldots, 4)$ and $B(=D)$ are given in Table II. Therefore, the combined T-S fuzzy models which approximate $\Sigma_{\bar{V}}$ is in the form of (8).

TABLE I

THE SMART AIRFOIL MODEL PARAMETERS AND THEIR VALUES

\begin{tabular}{cc}
\hline Parameter & Value \\
\hline$b$ & $\frac{5}{12}(\mathrm{ft})$ \\
$\bar{e}$ & 0.35 \\
$\bar{g}$ & 0.0188 \\
$\bar{V}_{m}$ & 2.00 \\
$\bar{V}_{M}$ & 2.98 \\
$\bar{x}_{\alpha}$ & 0.25 \\
$\bar{r}_{\alpha}$ & 0.6229 \\
$\beta$ & 0.1 \\
$\mu$ & 152 \\
$\omega_{\alpha}$ & $64.1(\mathrm{rad} / \mathrm{s})$ \\
$\omega_{h}$ & $55.9(\mathrm{rad} / \mathrm{s})$ \\
\hline
\end{tabular}

The T-S fuzzy model approximation is validated by comparing the numerical simulation between the developed T-S fuzzy model and the aeroservoelastic model (2) with given initial condition, $x_{0}=\left[\begin{array}{llll}0 & 0 & 0.1 & 0\end{array}\right]$, and zero input/disturbance. For illustration, Fig. 2 shows the validation results for aerodynamic states $\alpha$ and $\dot{\alpha}$ when $\bar{V}=2.92$. It can be seen that the T-S model and the aeroservoelastic model are indistinguishable. Though not reported here, we have also validated T-S fuzzy models at various $\bar{V}$ within the range of consideration and they were all well matched with the corresponding aeroservoelastic models. We assume the nondimensional control input amplitude and rate bounds (3- $\sigma$ ) to be 1 and 300, respectively, and the random disturbance $d$ has intensity of 0.05 .

The T-S fuzzy full-state feedback control gains can be obtained by solving Eqs. (31)-(35) in Theorem 1. As an
TABLE II

THE T-S FUZZY MODEL PARAMETERS AND MATRICES

\begin{tabular}{|c|c|c|c|c|}
\hline Matrix & \multicolumn{4}{|c|}{ Value } \\
\hline$A_{1}$ & {$\left[\begin{array}{c}0 \\
-0.8959 \\
0 \\
0.5772\end{array}\right.$} & $\begin{array}{c}1 \\
-0.0555 \\
0 \\
0.0704\end{array}$ & $\begin{array}{c}0 \\
0.2185 \\
0 \\
-0.9757\end{array}$ & $\left.\begin{array}{l}0 \\
0 \\
1 \\
0\end{array}\right]$ \\
\hline$A_{2}$ & {$\left[\begin{array}{c}0 \\
-0.8959 \\
0 \\
0.5772\end{array}\right.$} & $\begin{array}{c}1 \\
-0.0555 \\
0 \\
0.0704\end{array}$ & $\begin{array}{c}0 \\
0.2185 \\
0 \\
-1.0933\end{array}$ & $\left.\begin{array}{l}0 \\
0 \\
1 \\
0\end{array}\right]$ \\
\hline$A_{3}$ & {$\left[\begin{array}{c}0 \\
-0.8959 \\
0 \\
0.5772\end{array}\right.$} & $\begin{array}{c}1 \\
-0.0555 \\
0 \\
0.0704\end{array}$ & $\begin{array}{c}0 \\
0.1258 \\
0 \\
-0.9757\end{array}$ & $\left.\begin{array}{l}0 \\
0 \\
1 \\
0\end{array}\right]$ \\
\hline$A_{4}$ & {$\left[\begin{array}{c}0 \\
-0.8959 \\
0 \\
0.5772\end{array}\right.$} & $\begin{array}{c}1 \\
-0.0555 \\
0 \\
0.0704\end{array}$ & $\begin{array}{c}0 \\
0.1258 \\
0 \\
-1.0933\end{array}$ & $\left.\begin{array}{l}0 \\
0 \\
1 \\
0\end{array}\right]$ \\
\hline$B=D$ & & $\begin{array}{c}0 \\
-0.1428 \\
0 \\
0.5767\end{array}$ & $\times 10^{-3}$ & \\
\hline
\end{tabular}
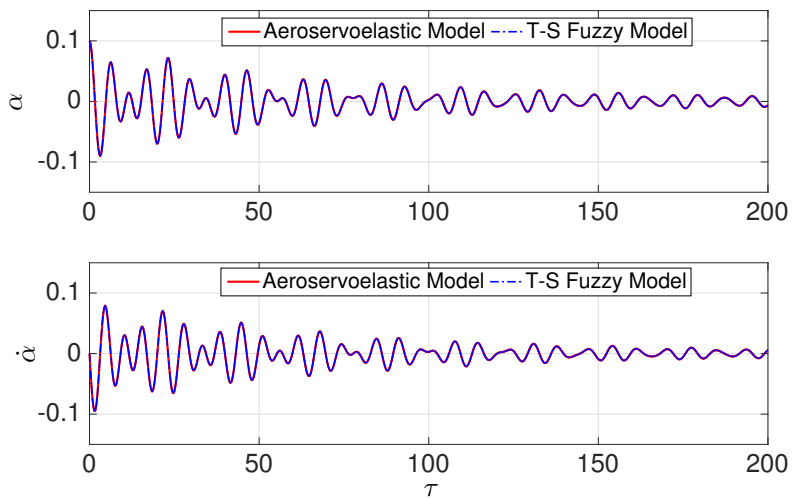

Fig. 2. T-S fuzzy model validation for $\alpha$ and $\dot{\alpha}$ when $\bar{V}=2.92$.

illustration, Fig. 3 shows the comparison between the openloop and closed-loop response of aerodynamic states $\alpha$ and $\dot{\alpha}$ when $\bar{V}=2.92$. As can be seen, the motion of ballast mass is able to suppress the airfoil vibration effectively, and the control input and input rate are within their $(3-\sigma)$ bounds, as shown in Fig. 4. The corresponding feedback gains for augmented T-S model are listed in Table III.

\section{CONCLUSIONS}

In this paper, we presented a new approach to address the problem of optimal variance control for a smooth nonlinear dynamic system subject to actuator amplitude and rate constraints. The convexity property of the T-S fuzzy model was used to approximate such nonlinear system via a convex 
TABLE III

T-S FUZZY FULL-STATE FEEDBACK GAINS WHEN $\bar{V}=2.92$

\begin{tabular}{cc}
\hline Gains & Value \\
\hline$F_{1}$ & {$[-17.7546,27.2523,-21.5733,9.1304,0.1694]$} \\
$F_{2}$ & {$[-17.5771,27.3676,-23.9292,14.3077,0.1713]$} \\
$F_{3}$ & {$[-19.6901,42.1545,-26.6340,7.1636,0.1474]$} \\
$F_{4}$ & {$[-20.1295,51.0647,-27.2346,18.6509,0.1358]$} \\
\hline
\end{tabular}
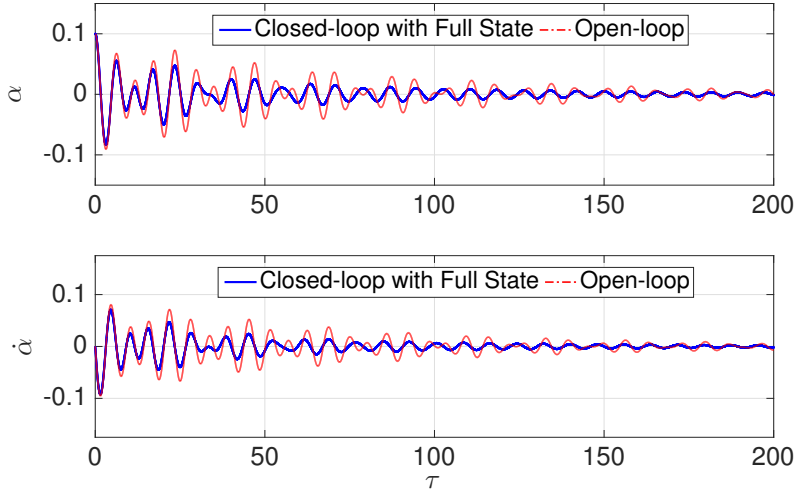

Fig. 3. Open-loop and closed-loop response comparison when $\bar{V}=2.92$.
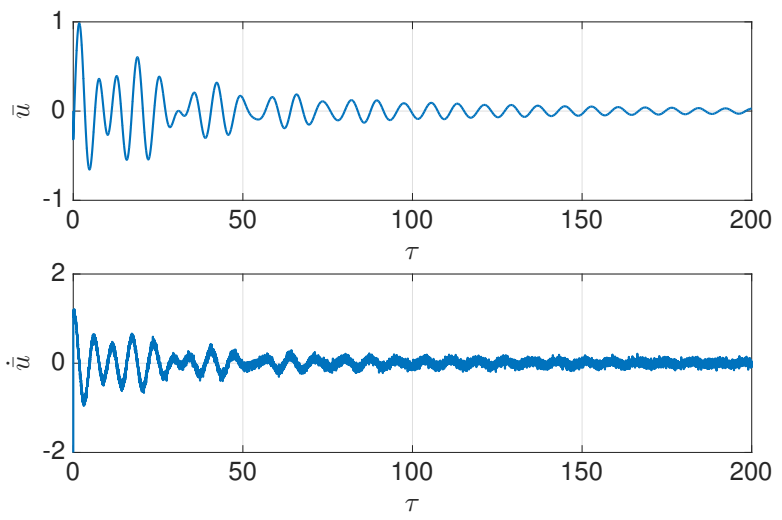

Fig. 4. Control input and input rate response when $\bar{V}=2.92$.

combination of local linear models. The original boundedinput and bounded-input rate problem was converted into a bounded-output and bounded-input problem by augmenting the control input as a new state. This was then formulated using the LMIs by utilizing the solutions to the input and output variance constraint control problems. A fuzzy dynamic full-state feedback controller was developed based on a fuzzy static full-state feedback controller that was designed for the augmented system. This controller was then applied to controlling a smart airfoil model. The simulation results confirmed that the proposed fuzzy full-state feedback controller was effective in flutter suppression over a given range of velocity.

\section{ACKNOWLEDGMENT}

The authors would like to thank Professor G. Zhu of Michigan State University and Dr. Nhan Nguyen of NASA
Ames Research Center for their kind support and helpful discussions. This research was supported by NASA ARMD Convergent Aeronautics Solutions (CAS) Project. The work of the second author was supported by the Summer Faculty Fellowship Program at NASA Ames Research Center.

\section{REFERENCES}

[1] J. M. Shewchun, E. Feron, "High performance bounded control of systems subject to input and input rate constraints," AIAA Guidance, Navigation, and Control Conference, New Orleans, LA, 1997.

[2] R. A. Hess and S. A. Snell, "Flight control system design with rate saturating actuators," J. of Guidance, Control, and Dynamics, vol. 20, no. 1, pp. 90-96, 1997.

[3] Z. Lin, "Semi-global stabilization of linear systems with position and rate-limited actuators," Systems \& Control Letters, vol. 30, pp. 1-11, 1997.

[4] S. A. Snell and R. A. Hess, "Robust, decoupled flight control design with rate-saturating actuators," Journal of Guidance, Control, and Dynamics, vol. 21, No. 3, pp.361-367, 1998.

[5] I. Emre Kose and F. Jabbari, "Control of systems with actuator amplitude and rate constraints," Proceedings of the American Control Conference, Arlington, VA, 2001.

[6] F. Wu, "A generalized LPV system analysis and control synthesis framework," International Journal of Control, 74(7), pp. 745-759, 2001.

[7] R. C. L. F. Oliveira and P. L. D. Peres, "Time-varying discrete-time linear systems with bounded rates of variation: stability analysis and control design," Automatica, 45(11), pp. 2620-2626, 2009.

[8] T. Takagi and M. Sugeno, "Fuzzy identification of systems and its applications to modeling and control," IEEE Transactions on Systems, Man, and Cybernetics, vol. 15, pp. 116-132, 1985.

[9] H. O. Wang, K. Tanaka, and M. Griffin, "An approach to fuzzy control of nonlinear systems: stability and design issues," IEEE Transactions on Fuzzy Systems, vol. 4, pp. 14-23, 1996.

[10] K. Tanaka and M. Sugeno,"Stability analysis of fuzzy systems using Lyapunov's direct method," Proceedings of NAFIPS90, University of Toronto, Toronto, pp. 133-136, 1990.

[11] K. Tanaka and H. O. Wang, Fuzzy control systems design and analysis: A linear matrix inequality approach, John Wiley \& Sons, 2001.

[12] S. M. Swei and Y. T. Jiang, "On the efficiency of the smart airfoil model in active flutter suppression," Proceedings of $2^{\text {nd }}$ International Conference on Motion and Vibration Control, Yokohama, Japan, pp. 593-598, 1994.

[13] A. White, G. Zhu, and J. Choi, "A linear matrix inequality solution to the output covariance constraint control problem," ASME Dynamic Systems and Control Conference, vol. 3, pp. 163-169, doi:10.1115/DSCC2012MOVIC2012-8799.

[14] S. G. Cao, N.W. Rees, and G. Feng, "Fuzzy control of nonlinear continuous-time systems," $35^{\text {th }}$ IEEE Conference on Decision and Control, Kobe, Japan, pp. 592-597, 1996.

[15] R. E. Skelton, Dynamic systems control: Linear systems analysis and synthesis, John Wiley \& Sons, 1988.

[16] M. Corless, G. Zhu, and R. E. Skelton, "Robustness of covariance controllers," $10^{\text {th }}$ Allerton Conference on Communication, Control and Computing, Monticello, IL, 1989.

[17] H. O. Wang, K. Tanaka, and M. Griffin,“ Parallel distributed compensation of nonlinear systems by Takagi-Sugeno fuzzy model," $4^{\text {th }}$ IEEE International Conference on Fuzzy Systems and $2^{\text {nd }}$ International Fuzzy Engineering Symposium, Yokohama, Japan, vol. 2, pp. 531-538, 1995. 